\title{
High Accuracy Many-Body Calculational Approaches for Excitations in Molecules
}

\author{
Jeffrey C. Grossman ${ }^{1, \dagger}$, Michael Rohlfing ${ }^{2}$, Lubos Mitas ${ }^{3}$, Steven G. Louie ${ }^{1}$, and Marvin L. Cohen ${ }^{1}$ \\ ${ }^{1}$ Department of Physics, University of California at Berkeley and Materials Sciences Division, Lawrence Berkeley National \\ Laboratory, Berkeley, CA 94720. \\ ${ }^{2}$ Institut für Theoretische Physik II - Festkörperphysik, Universität Münster, 48149 Münster, Germany \\ ${ }^{3}$ National Center for Supercomputing Applications, University of Illinois at Urbana-Champaign, Urbana, Illinois 61801
}

(October 30, 2018)

\begin{abstract}
Two state-of-the-art computational approaches: quantum Monte Carlo (QMC), based on accurate total energies, and GW with exciton effects (GW-BSE), based on perturbation theory are employed to calculate ionization potentials, electron affinities, and first excited singlet and triplet energies for the silane and methane molecules. Results are in excellent agreement between these dramatically different approaches and with available experiment. The optically forbidden triplet excitation in silane is predicted to lie roughly $1 \mathrm{eV}$ higher than previously reported. For methane, the impact of geometry relaxation is shown to be $\sim 2 \mathrm{eV}$ for excited states. Further, in the GW-BSE method, we demonstrate that inclusion of off-diagonal matrix elements in the self-energy operator is crucial for an accurate picture.
\end{abstract}

Optical excitations play a fundamental role in technological applications such as dye chemicals, photovoltaics, laser technology, and catalysts for chemical reactions. An accurate evaluation of excited states requires more sophisticated approaches than simply a straightforward application of the mainstream ground state methods such as density functional theory and Hartree-Fock. In addition, many traditional approaches for calculating excited states can be limited by a range of factors. For example, configuration interaction with single excitations is missing correlation effects and is often inaccurate, optical transitions modeled as quasiparticle energies corrected by excitonic effects rely on an approximate description of the electron-hole interaction, and basis set correlated wave function approaches are limited by their scaling with the number of correlated electrons.

Two promising new approaches have been developed to calculate accurate excitation energies: (1) quantum Monte Carlo (QMC), based on a stochastic solution to the many-electron Schrödinger equation, can provide chemical accuracy (i.e., $0.05 \mathrm{eV}$ ) for the total energy while at the same time scales as $\mathrm{N}^{3}$ where $\mathrm{N}$ is the number of valence electrons (see, e.g., [1],2]), and (2) many-body Green's function perturbation techniques that employ the $G W$ approximation [3, [1] for the electron self-energy operator to calculate the single-particle excitation spectrum, followed by a solution to a Bethe-Salpeter equation [5] (BSE) for electron-hole excitations [6].

In this Letter, we demonstrate excellent agreement between QMC, GW-BSE, and available experiment for excitation energies of silane $\left(\mathrm{SiH}_{4}\right)$ and methane $\left(\mathrm{CH}_{4}\right)$. Calculated ionization potentials, electron affinities, and first excited singlet and triplet energies also agree very well with highly reliable standard quantum chemistry approaches; however, the major advantage of our two current approaches is numerical efficiency. Both methods require moderate computation times, and their scaling with system size is to our knowledge superior to all other quantum-chemistry approaches for computing excited states. The excellent agreement between QMC and GW-BSE is encouraging given the dramatically different methodologies involved (i.e., the former relies on accurate total energy differences while the latter is based on perturbation theory).

In our QMC approach [7 11, variational Monte Carlo is employed to find an optimized correlated many-body trial function. This trial function, $\psi_{T}(R)$, is a product of Slater determinants, $D_{n}$, and a correlation factor [12],

$$
\psi_{T}=\sum_{n} d_{n} D_{n}^{\uparrow} D_{n}^{\downarrow} \exp \left[\sum_{I, i<j} u\left(r_{i I}, r_{j I}, r_{i j}\right)\right],
$$

where $I$ corresponds to the ions, $i, j$ to the electrons and $r_{i I}, r_{j I}, r_{i j}$ to the relevant separations. Parametrization and optimization of $u\left(r_{i I}, r_{j I}, r_{i j}\right)$, which represents the electron-electron and electron-electron-ion correlations, is described in Ref. 13]. In the Slater determinant part, we employ natural orbitals rather than HartreeFock (HF) or density functional orbitals [8]. To eliminate most of the remaining variational bias we use the fixed node diffusion Monte Carlo method, which is based on the property that the operator $\exp (-\tau H)$, where $H$ is the Hamiltonian, projects out the ground state of any trial function with the same symmetry and non-zero overlap, subject to the constraint that the nodes are unchanged [14. All QMC results presented here are from the diffusion Monte Carlo approach.

The GW-BSE Green's function perturbation approach consists of three successive steps. In the first step a local density approximation (LDA) 15 calculation is performed to obtain the electronic ground state. In the second step the LDA results are used to construct the electronic self-energy operator $\Sigma$ (also known as the mass operator). This is done within the $G W$ approximation 3, 11 where $G$ is the (LDA) single-particle Green's function and $W$ is the screened Coulomb interaction based on 
RPA dielectric screening. Solving the equation-of-motion for the single-particle Green's function yields the quasiparticle states (i.e., the occupied (hole) states $v$ and the empty (electron) states $c$ ) of the molecule. In the third step the electron-hole interaction $\left\langle v c\left|K^{\mathrm{eh}}\right| v^{\prime} c^{\prime}\right\rangle$ between the occupied states $v$ and the empty states $c$ is evaluated. The coupled electron-hole excitations $|S\rangle=\sum_{v c} A_{v c}^{S}|v c\rangle$ and their excitation energies $\Omega^{S}$ then result from the Bethe-Salpeter equation

$$
\left(\varepsilon_{c}^{\mathrm{QP}}-\varepsilon_{v}^{\mathrm{QP}}\right) A_{v c}^{S}+\sum_{v^{\prime} c^{\prime}}\left\langle v c\left|K^{\mathrm{eh}}\right| v^{\prime} c^{\prime}\right\rangle A_{v^{\prime} c^{\prime}}^{S}=\Omega^{S} A_{v c}^{S} .
$$

For details, we refer to Refs. [6, 16].

We have also calculated excited state energies as total energy differences using LDA and the generalized gradient approximation (GGA) with the PW91 functional [17]. In addition, the small number of correlated electrons in the present systems permits us to employ several of the most accurate quantum chemical approaches: coupled cluster with single, double, and perturbationally triple $(\operatorname{CCSD}(\mathrm{T}))$ excitations [18], and complete active space self consistent field (CASSCF) with an active space of 8 electrons and 9 active orbitals. For these methods, extensive basis set tests showed that energy differences are converged to better than $0.02 \mathrm{eV}$ with the cc-pVQZ 119 basis set. All calculations presented here other than QMC and GW-BSE have been carried out using the G98 package [20] and the cc-pVQZ basis set.

It is important to distinguish between the two different methodologies employed here. Using total energy methods, the ionization potential (IP) and electron affinity (EA) are computed as total energy differences. In the Green's function, the QP energies are obtained in a different manner, namely as a solution of a quasiparticle Dyson's equation [6].

The single-particle HF and LDA energy spectra for $\mathrm{SiH}_{4}$ are shown in Fig. 11. While both methods are in qualitative agreement with one another, there is a large quantitative discrepancy, as expected: HF usually overestimates HOMO-LUMO gaps and eigenvalue spacings, whereas LDA typically underestimates these quantities. Note that since the first excitations are from $t_{2}$ to $a_{1}$ orbitals, the ground and first excited singlet states of $\mathrm{SiH}_{4}$ are of different symmetry.

It is reassuring that all of the methods (see Table $\mathbb{1}$ ) are in good agreement with experiment for the ionization potential of $\mathrm{SiH}_{4}$. The LDA, GGA, and HF results are $\sim 0.5 \mathrm{eV}$ lower than experiment, indicating a slight bias towards the ionized state; however, note the dramatic improvement when compared to their single-particle highest occupied eigenvalue. The calculations indicate a small, negative (i.e., nonphysical) electron affinity ranging from -0.1 to $-0.6 \mathrm{eV}$.

Within the GW-BSE approach, the behavior of the $a_{1}$
LUMO state deserves careful discussion (see Table [1]). In the underlying LDA calculation, the LUMO energy is below the vacuum level (see Fig. 1) and is thus given by a localized, bound wave function. Within first-order perturbation treatment of the self-energy operator $\Sigma$, the orbitals are kept as LDA orbitals and the LUMO energy is shifted above the vacuum level by $1.7 \mathrm{eV}$ (see second column of Tab. [1]). Being unbound now, the QP state changes its wave function from a localized to a more extended orbital. This is described by going beyond the diagonal evaluation of $\Sigma$ in the LDA basis in the $G W$ calculation and taking off-diagonal elements of $\Sigma$ into account, which are in fact non-zero. This effect, which was not accounted for in a previous work [6], lowers the LUMO state by as much as $0.8 \mathrm{eV}$ (see third column of Tab. III. The LUMO energy of $0.3 \mathrm{eV}$ is now in good agreement with the results of the other methods. Note that the LUMO state (being unbound) converges slowly with respect to the basis, due to its delocalized nature. The low-energy electron-hole excited states, on the other hand, that are the focus of this paper, are spatially localized since the electron is attracted to the hole; these excited states are much easier to converge.

Our results for $\mathrm{SiH}_{4}$ excitation energies for the lowestenergy triplet and singlet excited states are given in Table III. Calculation of spin-singlet excitations by total energy differences poses a serious challenge to traditional approaches. For example, the first excited singlet state is described by a 2 determinant wavefunction. Accurate correlation of this state was therefore only possible using the CASSCF method. The triplet state is significantly easier to converge within traditional approaches owing to the fact that it is the lowest energy state of the given spin multiplicity. The QMC approach does not encounter the same difficulties as the traditional approaches for the singlet state since the spin configuration is given by specification of the slater determinant - singlet and triplet states are equally as simple to simulate, provided each wavefunction is orthogonal to the ground state. In the case of the excited singlet state, the DMC energy was found to be rather insensitive (i.e., $<0.1 \mathrm{eV}$ ) to the number of determinants in the trial function, indicating that the nodes of a single determinant closely resemble those of the 2-determinant wavefunction which has the correct spatial symmetry.

The $\mathrm{SiH}_{4}$ spin singlet results are in good agreement with experiment 21 for the three theoretical methods listed in Table III. The optically forbidden triplet excitation is difficult to obtain from experiments. Using electron collision spectroscopy, slight spectral structures were found at around 7-8 eV [22]. We believe that the agreement among the theoretical methods presented here strongly suggests that the triplet excitation lies roughly $1 \mathrm{eV}$ higher than previously reported.

The GW-BSE results of Table III differ from the ones of Ref. [6] (see also Table II). The reason is again due 
to the off-diagonal matrix elements of the self-energy operator when expanded in the LDA orbitals, which had been neglected in Ref. [6]. As discussed above, the full self-energy operator allows the unoccupied states to become more delocalized. Therefore the overlap between the hole and electron states, as well as the electron-hole interaction, is significantly reduced. Concomitantly, the excitation energies (column 3 in Tab. III) are not reduced compared to column 2 (as the lower LUMO energy suggests), but are in fact increased. It is interesting to note that the triplet excitation energy is more affected than the singlet, which reduces the singlet-triplet splitting from $1.1 \mathrm{eV}$ to $0.7 \mathrm{eV}$ within GW-BSE.

Methane and silane are isoelectronic and similar in their valence shell structure; however, the stronger potential of carbon compared to silicon causes wavefunctions and densities to be less smooth which in turn can lead to difficulties and inaccuracies within ab initio calculations. We have carried out the same calculations described above for $\mathrm{CH}_{4}$. Our results, shown in Table IV, again demonstrate strong agreement between QMC and GW-BSE.

An important issue in the discussion of molecular spectra is the interaction between electronic excitations and the molecular geometry. Despite the high dimensionality of a five-atom molecule, we are able to discuss the most important aspects of geometric relaxation in the excited states and their consequences for the total energy. Table IV illustrates the impact of relaxation from the ground state $\mathrm{T}_{d}$ symmetry for each excited state molecule. Note that the optimization has been restricted to $\mathrm{C}_{2 v}$ symmetry. Test calculations show that further lowering the symmetry results in a total energy reduction no larger than $\sim 0.1 \mathrm{eV}$.

For all three excited states shown here, geometry relaxation yields an enormous lowering of the total energy by as much as $1.9 \mathrm{eV}$. A minor contribution (about 0.2 $0.4 \mathrm{eV}$ ) is related to the increase of the $\mathrm{C}-\mathrm{H}$ distance in the excited states. The main contribution results from breaking the $T_{d}$ symmetry of the ground state. This effect can be understood from the molecular orbital scheme (see Fig. 1). Within the ground state, all three degenerate HOMO levels are fully occupied, i.e. the ground state is non-degenerate. In the excited states, one of the three HOMO states becomes half-filled, i.e. the excited states are three-fold degenerate. The symmetry-breaking from $T_{d}$ to $C_{2 v}$ lifts the degeneracy of the three HOMO levels. In the single-particle spectrum of Fig. 1 two HOMO levels are shifted down and one is shifted up, forming the new HOMO level of the molecule in the reduced symmetry. In the optimum geometry of the excited states this level-splitting is about $4.2 \mathrm{eV}$, although the centerof-mass of the three levels remains roughly the same. The new HOMO level, which is half-filled in all three excited states, is non-degenerate, so the excited states are nondegenerate as well.
A closer analysis of the excited-state geometry shows that the increase of the $\mathrm{C}-\mathrm{H}$ bond length is relatively small (no more than $0.07 \AA$ ). The main effect results indeed from the Jahn-Teller distortion of the molecule, which changes the bond angles from 109.47 degree (tetrahedral angle) to 94 degree for the small and 118 degree for the large bond angles. This distortion increases the total energy of the ground state by $1.5 \mathrm{eV}$. In the excited states, however, this increase of the total energy is overcompensated by the splitting of the three HOMO levels described above, i.e. by the upwards shift of the new HOMO level and a correspondingly large reduction of the excitation energies. In the experimental spectrum for $\mathrm{CH}_{4}$ 23], a weak shoulder is observed at about $10 \mathrm{eV}$, corresponding to a vertical excitation. Compared with the onset at $8.52 \mathrm{eV}$, this indicates a relaxation gain of about $1.5 \mathrm{eV}$, supporting our calculated results.

Similar effects on the excitation energy are also found for $\mathrm{SiH}_{4}$. The gain in total energy is $0.9 \mathrm{eV}$, i.e. slightly smaller than in $\mathrm{CH}_{4}$. In the measured absorption spectrum this corresponds to the difference between the energy of maximum absorption and the low-energy onset. This difference amounts to $0.6 \mathrm{eV}$ in the absorption measurements of Ref. 21. Our minimum-energy transition for $\mathrm{SiH}_{4}$ is $8.3 \mathrm{eV}$ (in GW-BSE), in excellent agreement with the measured onset of the spectrum at $8.2 \mathrm{eV}$.

JCG acknowledges useful discussions with G.M. Rignanese and E. Chang. Support was provided by National Science Foundation Grant No. DMR-9520554 and by the Director, Office of Energy Research, Office of Basic Energy Sciences, Materials Sciences Division of the U. S. Department of Energy under Contract No. DE-AC0376SF00098. MR acknowledges financial support by the Deutsche Forschungsgemeinschaft (Bonn, Germany) under grant No. Ro 1318/2-1. Computational resources have been provided by NCSA and by NERSC.

$\dagger$ Present Address: Lawrence Livermore National Laboratory, 7000 East Avenue, L-415, Livermore, CA 94550.

[1] J. C. Grossman and L. Mitas, Phys. Rev. Lett. 79, 4353 (1997).

[2] W. A. Lester, ed. Recent Advances in Quantum Monte Carlo Methods (World Scientific, Singapore, 1997).

[3] L. Hedin and S. Lundqvist, Solid State Physics 23, 1 (1969).

[4] M.S. Hybertsen and S.G. Louie, Phys. Rev. Lett. 55, 1418 (1985); Phys. Rev. B 34, 5390 (1986).

[5] G. Strinati, Phys. Rev. B 29, 5718 (1984).

[6] M. Rohlfing and S.G. Louie, Phys. Rev. Lett. 80, 3320 (1998). 
[7] L. Mitas, E. L. Shirley, and D. M. Ceperley, J. Chem. Phys. 95, 3467 (1991).

[8] J. C. Grossman and L. Mitas, Phys. Rev. Lett. 74, 1323 (1995).

[9] B. L. Hammond, W. A. Lester, Jr., and P. J. Reynolds, Monte Carlo Methods in Ab Initio Quantum Chemistry, World Scientific, 1994.

[10] D. M. Ceperley and L. Mitas, Quantum Monte Carlo Methods in Chemistry, Advances in Chemical Physics, Vol. XCIII, John Wiley, 1996.

[11] S. Fahy, X. W. Wang, and S. G. Louie, Phys. Rev. Lett. 61, 1631 (1988).

[12] K. E. Schmidt and J. W. Moskowitz, J. Chem. Phys. 93, 4172 (1990).

[13] L. Mitas, Pseudopotential quantum Monte Carlo for large-z atom systems, in Computer Simulation Studies in Condensed-Matter Physics V, edited by D. Landau, K. Mon, and H.-B. Schuttler, Springer Proceedings in Physics, Springer-Verlag, Berlin, 1993.

[14] W.M.C. Foulkes, R.Q. Hood, and R.J. Needs, Phys. Rev. B 60, 4558 (1999).

[15] P. Hohenberg and W. Kohn, Phys. Rev. 136, B864 (1964); W. Kohn and L. J. Sham, Phys. Rev. 140, A1133 (1965).

[16] M. Rohlfing and S.G. Louie, to be published.

[17] J. P. Perdew, in Electronic Structure of Solids '91, edited by P. Ziesche and H. Eschrig, page 11, Akademie Verlag, Berlin, 1991.

[18] K. Raghavachari and J. B. Anderson, J. Phys. Chem. 100, 12960 (1996).

[19] T. H. Dunning, Jr., J. Chem. Phys. 90, 1007 (1989).

[20] M. J. Frisch, et. al., GAUSSIAN94, Revision B.1 (Gaussian Inc., Pittsburgh, PA, 1995.

[21] U. Itoh, Y. Toyoshima, and H. Onuki, J. Chem. Phys. 85, 4867 (1986).

[22] H. Tanaka, L. Boesten, M. Kimura, M.A. Dillon, and D. Spence, J. Chem. Phys. 92, 2115 (1990).

[23] G. Herzberg, Molecular Spectra and Molecular Structure, Vol. III: Electronic Spectra and Electronic Structure of Polyatomic Molecules (Van Nostrand, New York 1966).

FIG. 1. Single-particle Hartree-Fock and local density approximation eigenvalue spectra $(\mathrm{eV})$ for the $\mathrm{SiH}_{4}$ molecule. Note that the $\mathrm{CH}_{4}$ molecule has the same orbital structure.

TABLE I. Ionization potential (IP) and quasiparticle (QP) gap $(\mathrm{eV})$ for $\mathrm{SiH}_{4}$, computed as $\mathrm{E}(\mathrm{N}-1)-\mathrm{E}(\mathrm{N})$ and $\mathrm{E}(\mathrm{N}+1)+$ $\mathrm{E}(\mathrm{N}-1)-2 \mathrm{E}(\mathrm{N})$, respectively, for all methods except GW.

\begin{tabular}{lll}
\hline \hline & IP & QP Gap \\
\hline HF & 12.3 & 12.6 \\
LDA & 12.1 & 12.4 \\
BPW91 & 12.1 & 12.5 \\
CCSD(T) & 12.7 & 13.3 \\
GW & 12.7 & 13.0 \\
QMC & $12.6(1)$ & $12.8(1)$ \\
EXP $^{a}$ & 12.6 & - \\
\hline \hline
\end{tabular}

${ }^{a}$ Ref. 21.

TABLE II. Quasiparticle levels and lowest electron-hole excitation energies (eV), calculated for $\mathrm{SiH}_{4}$ within LDA and within two evaluations of the GW-BSE approach. Unlike the second column, the GW-BSE results of the third column includes off-diagonal matrix elements of the self-energy operator and allows for changes in the one-particle wave functions.

\begin{tabular}{lrrr}
\hline \hline & LDA & GW-BSE (diagonal $\Sigma$ ) & GW-BSE (full $\Sigma$ \\
\hline HOMO energy & -8.4 & -12.7 & -12.7 \\
LUMO energy & -0.6 & 1.1 & 0.3 \\
$E_{S}$ & 7.8 & 8.8 & 9.2 \\
$E_{T}$ & 7.8 & 7.7 & 8.5 \\
\hline \hline
\end{tabular}

TABLE III. Neutral excitation energies (eV) for the first excited singlet and triplet states of $\mathrm{SiH}_{4}$ computed as $\mathrm{E}$ (excited state) - E(ground state) for all methods except GW-BSE. $\Delta$ corresponds to the singlet-triplet splitting. The experimental result 21] denotes the energy of maximum absorption for the state studied here, which corresponds to vertical excitation.

\begin{tabular}{llll}
\hline \hline & singlet & triplet & $\Delta$ \\
\hline HF & - & 8.4 & - \\
LDA & - & 8.1 & - \\
BPW91 & - & 8.2 & - \\
CCSD(T) & - & 8.7 & - \\
CASSCF & 9.1 & 8.7 & 0.4 \\
GW-BSE & 9.2 & 8.5 & 0.7 \\
QMC & $9.1(1)$ & $8.7(1)$ & $0.4(1)$ \\
EXP & 8.8 & - & - \\
\hline \hline
\end{tabular}

TABLE IV. Ionization potentials (IP) and energies of the lowest singlet $\left(\mathrm{E}_{S}\right)$ and triplet $\left(\mathrm{E}_{T}\right)$ excited states for $\mathrm{CH}_{4}$ in both the ground state $\left(\mathrm{T}_{d}\right)$ and relaxed $\left(\mathrm{C}_{2 v}\right)$ symmetries. All energies are in $\mathrm{eV}$ and are given with respect to the ground state. The experimental result 23] denotes the onset of the spectrum, which corresponds to the minimum energy transition including structural relaxation.

\begin{tabular}{llll}
\hline \hline & IP & $\mathrm{E}_{T}$ & $\mathrm{E}_{S}$ \\
\hline GW-BSE $\left(\mathrm{T}_{d}\right)$ & 14.3 & 10.1 & 10.5 \\
QMC $\left(\mathrm{T}_{d}\right)$ & $14.3(1)$ & $10.1(1)$ & $10.4(1)$ \\
GW-BSE $\left(\mathrm{C}_{2 v}\right)$ & 12.5 & 8.2 & 8.6 \\
QMC $\left(\mathrm{C}_{2 v}\right)$ & $12.7(1)$ & $8.4(1)$ & $8.7(1)$ \\
EXP & $12.99^{a}$ & - & 8.52 \\
\hline \hline
\end{tabular}

${ }^{a}$ Ref. 23. 


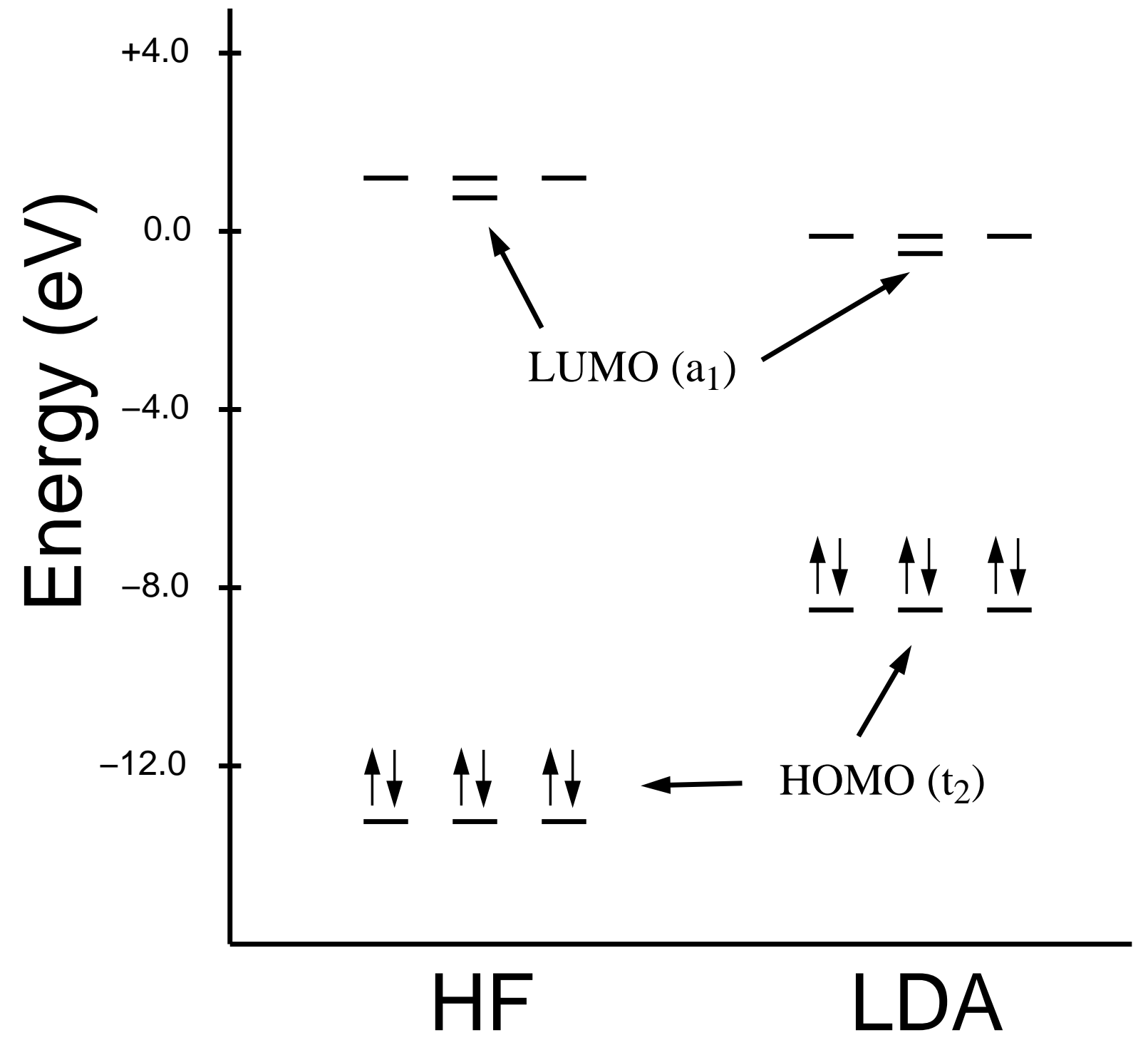

